\title{
COMPARISON OF THREE SLEEP QUESTIONNAIRES IN SCREENING OBSTRUCTIVE SLEEP APNOEA
}

\author{
Preethi Perumalsamy ${ }^{1}$, Arvin Kumar Charles², Gangi Reddy3, Chandrasekar Chokkalingam4, Rajagopalan Balakrishnan 5 \\ $13^{\text {rd }}$ Year Postgraduate Student, Department of Chest and TB, Sri Ramachandra Medical Hospital, Chennai. \\ ${ }^{2}$ Bachelor of Sciences (Allied Health Sciences), Department of Chest and TB, Sri Ramachandra Medical Hospital, Chennai. \\ ${ }^{3}$ Assistant Professor, Department of Chest and TB, Sri Ramachandra Medical Hospital, Chennai. \\ ${ }^{4}$ Professor, Department of Chest and TB, Sri Ramachandra Medical Hospital, Chennai. \\ ${ }^{5}$ Professor, Department of Chest and TB, Sri Ramachandra Medical Hospital, Chennai.
}

ABSTRACT
BACKGROUND
Obstructive sleep apnoea (OSA) is a sleep-related breathing disorder characterised by full or partial occlusion of the upper airway
during sleep. Obstructive sleep apnoea (OSA) is a common disorder affecting at least $2 \%$ to $4 \%$ of the adult population and can
occur in all age groups.
The aim of our study is to assess the usefulness of Berlin, STOP-BANG and Epworth questionnaires in evaluation of obstructive
sleep apnoea and to find specificity and sensitivity of Berlin, STOP-BANG and Epworth questionnaires in predicting diagnosis of
obstructive sleep apnoea.

\section{MATERIALS AND METHODS}

A cross-sectional study aimed at predicting high risk of OSA based on using three questionnaires in comparison to the objective assessment using the standard overnight attended PSG on all the recruited patients. The study was conducted over 62 patients at the sleep disorders laboratory of pulmonary medicine, Sri Ramachandra University Hospital, Porur, Chennai. Anthropometric measures including body weight, height, body mass index (BMI) and neck circumference (NC) as well as gender were documented for all patients.

\section{RESULTS}

The incidence of OSA in our study population was $87 \%$ of which percentage of population with mild, moderate and severe OSA were $24 \%, 20.9 \%$ and $41.9 \%$. The above results show that the Berlin questionnaire score is a better tool than the STOP-BANG and Epworth score, since the average accuracy of Berlin questionnaire was 66.65 .

\section{CONCLUSION}

The above results show that the Berlin questionnaire score is a better tool than the STOP-BANG and Epworth score, since the average accuracy of Berlin questionnaire was 66.65. Large prospective studies will be required to help establish the exact role of subjective Berlin, STOP-BANG and Epworth sleep screening questionnaires in evaluating patient with OSA.

\section{KEYWORDS}

Obstructive Sleep Apnoea, Sleep Study, Berlin Questionnaire, Epworth Questionnaire, STOP-BANG Score, AHI.

HOW TO CITE THIS ARTICLE: Perumalsamy P, Charles AK, Reddy G, et al. Comparison of three sleep questionnaires in screening obstructive sleep apnoea. J. Evolution Med. Dent. Sci. 2017;6(88):6132-6136, DOI: 10.14260/jemds/2017/1332

\section{BACKGROUND \\ Obstructive sleep apnoea (OSA) is a sleep-related breathing disorder characterised by full or partial occlusion of the upper airway during sleep.[1] Obstructive sleep apnoea (OSA) is a common disorder affecting at least $2 \%$ to $4 \%$ of the adult population and can occur in all age groups.[2-3] It is estimated that nearly $80 \%$ of men and $93 \%$ of women with moderate- to-severe sleep apnoea are undiagnosed.[4] \\ OSA can be effectively treated in many patients with continuous positive airway pressure (CPAP). ${ }^{[5-6]}$ Therefore, it is important that OSA should formally be diagnosed to identify patients who could benefit from CPAP. However, it is not easy to diagnose OSA, largely because of problems in accessibility.}

'Financial or Other Competing Interest': None.

Submission 26-08-2017, Peer Review 24-10-2017,

Acceptance 30-10-2017, Published 06-11-2017.

Corresponding Author:

Preethi Perumalsamy,

\#257/2, Sankarankovil Road,

$9^{\text {th }}$ Cross Street, Rajapalayam-626117,

Virudhunagar District, Tamilnadu.

E-mail: preethishasha@gmail.com

DOI: $10.14260 /$ jemds $/ 2017 / 1332$

\section{(c) (i) $\$$}

Although, the "gold standard" for diagnosis of OSA is laboratory polysomnography (PSG); however, the occurrence of OSA is far more prevalent than can be handled by the available sleep laboratories. Therefore, a screening tool is necessary to stratify patients based on their clinical symptoms, their physical examinations and their risk factors in order to ascertain patients at high risk and in urgent need of PSG and/or further treatment and patients at low risk who may not need PSG, but this is a cumbersome and expensive test.[7] A number of screening questionnaires and clinical screening models have been developed to help identify patients with OSA.[8-11]

This study aimed at comparing three established sleep questionnaires regarding their predictive probabilities for OSA.

\section{MATERIALS AND METHODS \\ Berlin Questionnaire}

The Berlin questionnaire has 11 questions grouped in 3 categories. The first category comprises of 5 questions concerning snoring, witnessed apnoeas and the frequency of such events. The second category comprises of 4 questions addressing daytime sleepiness with a sub-question about drowsy driving. The third category comprises of 2 questions 
concerning history of high blood pressure ( $>140 / 90 \mathrm{mmHg}$ ) and BMI of $>30 \mathrm{~kg} / \mathrm{m} 2$. Category 1 and 2 were considered positive if there was $\mathrm{P} 2$ positive responses to each category, while category 3 was considered positive with a self-report of high blood pressure and/or a BMI of $>30 \mathrm{~kg} / \mathrm{m} 2$. Study patients were scored as being at "high risk" of having OSA, if scores were positive for two or more of the three categories. Those patients who scored positively on less than two categories were identified as being at "low risk" of having OSA.

\section{Category 1}

Q1. Do you snore?

Yes (1)

No (0)

Do not know/refused

Q2. If you snore, your snoring is:

Slightly louder than breathing (0)

As loud as talking (0)

Louder than talking (1)

Very loud; can be heard in adjacent rooms

(1)

Do not know/ refused (0)

Q3. How often do you snore?

Nearly everyday (1)

3 to 4 nights per week (1)

1 to 2 nights per week (0)

1 to 2 nights per month (0)

Never or nearly never/ do not know $(0)$

Q4. Has your snoring ever bothered other people?

Yes (1)

No (0)

Do not know/ refused (0)

Q5. Has anyone noticed that you quit breathing during your sleep?

Nearly everyday (2)

3 to 4 times a week (2)

1 to 2 times a week (0)

1 to 2 times a month (0)

Never or nearly never/ do not know/refused (0)

\section{Category 2}

Q6. How often do you feel tired or fatigued after your sleep?

Nearly everyday (1)

3 to 4 times a week (1)

1 to 2 times a week (0)

1 to 2 times a month $(0)$

Never or nearly never/ do not

know/refused (0)

Q7. During your wake time do you

feel tired, fatigued or not up to par?

Nearly everyday (1)

3 to 4 times a week (1)

1 to 2 times a week (0)

1 to 2 times a month $(0)$

Never or nearly never/ do not

know/refused (0)

Q8. Have you ever nodded off or fallen asleep while driving a vehicle?

Yes (1)

No (0)

Do not know/ refused (0)

Q9. If yes, how often does it occur?

Nearly everyday (1)

3 to 4 times a week (1)

1 to 2 times a week (0)

1 to 2 times a month $(0)$

Never or nearly never/ do not

know/refused (0)
Category 3

Q10. Do you have high BP?

Yes (1)

No $(0)$

Do not know/ refused (0)

Q11. BMI, $\mathrm{kg} / \mathrm{m} 2$

$>30(1)$

$<30(0)$

High Risk of OSA Two or More Categories Scored as Positive: YES/ NO

Low Risk of OSA Less than Two Categories Scored as Positive: YES/ NO

\section{STOP-BANG Questionnaire}

The STOP-BANG Questionnaires consists of the following four questions:

S-- "Do you Snore loudly (louder than talking or loud enough to be heard through closed doors)?"

T-- "Do you often feel Tired, fatigued or sleepy during daytime?"

0-- "Has anyone Observed you stop breathing during your sleep?"

P-- "Do you have or are you being treated for high blood Pressure?"

BMI (BMI > $35 \mathrm{~kg} / \mathrm{m} 2)$,

Age $(>50$ years old),

Neck circumference $(\mathrm{NC}>40 \mathrm{~cm})$, and

Gender (Male) was termed the STOP-BANG questionnaire.

The answers to all questions of STOP-BANG questionnaire were designed in a simple yes/no format and the scores range from a value of 0 to 8 for STOP-BANG questionnaire, respectively. STOP-BANG questionnaire score subjects as either "high risk" or "low risk" for OSA. Answering yes to 3 or more questions in STOP-BANG questionnaire is considered "high risk," whereas answering yes to less than 3 questions in STOP-BANG questionnaire is considered "low risk." [12]

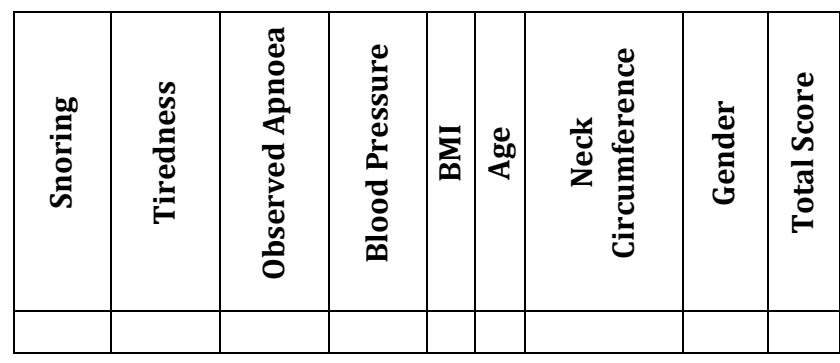

High Risk of OSA Answering Yes to three or more items: YES/ NO

Low Risk of OSA Answering Yes to less than three items: YES/ NO

\section{Epworth Sleepiness Scale}

The ESS is a self-administered questionnaire that asks subjects to rate how likely they would have dozed (fallen asleep) in 8 specific situations or activities that are commonly met in daily life. The chance of dozing is rated on a scale of 0 $30=$ would never dose, $1=$ slight chance of dozing, $2=$ moderate chance of dozing and $3=$ high chance of dozing). The total ESS score is the sum of 8-item scores and can range 
between 0 and 24 . The higher the score, the higher the person's level of daytime sleepiness, 11 - 24. Thus, the ESS final score was categorised into < 11 (low risk for sleepiness) and P11 (high risk for sleepiness).

\begin{tabular}{|c|c|}
\hline Situation & Chance of Dozing \\
\hline $\begin{array}{c}\text { Sitting in a relatively quiet place } \\
\text { and reading }\end{array}$ & 0123 \\
\hline Watching Television & 0123 \\
\hline $\begin{array}{c}\text { Sitting inactive in a public place } \\
\text { (e.g. a classroom, } \\
\text { meeting or theatre) }\end{array}$ & 0123 \\
\hline $\begin{array}{c}\text { As a passenger in a car or bus for an } \\
\text { hour without a break }\end{array}$ & 0123 \\
\hline Lying down to rest during the day & 0123 \\
\hline Sitting and talking to someone \\
\hline $\begin{array}{c}\text { Sitting quietly after lunch } \\
\text { (when you did not have alcohol) }\end{array}$ & 0123 \\
\hline $\begin{array}{c}\text { Stopping for a few minutes in the } \\
\text { traffic while driving }\end{array}$ & 0123 \\
\hline Total (Numerical Value) & \\
\hline
\end{tabular}

High risk of excessive daytime sleepiness ESS score $>11$ : YES/ NO

Low risk of excessive daytime sleepiness ESS score $<11$ : YES/ NO

\section{Polysomnography}

The diagnostic PSG was performed using computerised polysomnographic system (N4000 Embla, Somnologica, Iceland) including the monitoring of electroencephalogram (EEG), submental and anterior tibial electromyogram (EMG), oxygen saturation, electrocardiogram (ECG), inductance plethysmography of chest wall and abdomen, nasal pressure sensor and oronasal thermistor. The polysomnographic recording was scored manually by the sleep specialist, who was blinded to the results of the questionnaires and other clinical information concerning the patients. The sleep stage scoring and event scoring were done in accordance with the American Academy of Sleep Medicine (AASM) Manual for the Scoring of Sleep and Associated Events.

\section{Apnoea-Hypopnoea Index}

The severity of obstructive sleep apnoea is quantified by recording the number of pauses in breathing each hour that lasts longer than 10 seconds. This is referred to as the apnoea-hypopnoea index (AHI). Total obstructive apnoeahypopnoea index (AHI) was calculated as the number of obstructive apnoeas and hypopnoeas per hour of total sleep time (TST).

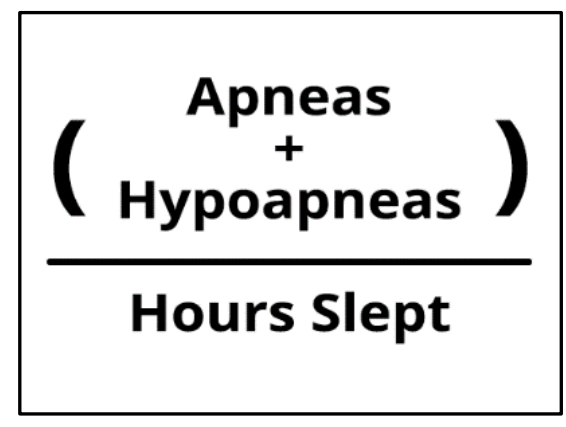

\begin{tabular}{|l|c|}
\hline \multicolumn{2}{|c|}{ Apnea Hypopnea Index (AHI) } \\
\hline Severity & AHI per Hour \\
\hline None/Minimal & $<5$ \\
Mild & $5-15$ \\
Moderate & $16-30$ \\
Severe & $>30$ \\
\hline
\end{tabular}

Research Methodology/ Study Design

This cross-sectional study aimed at predicting high risk of OSA based on using three questionnaires in comparison to the objective assessment using the standard overnight attended PSG on all the recruited patients.

All patients were interviewed by a sleep specialist and answered to the following three clinical questionnaires: Berlin, STOP-BANG and Epworth Sleepiness Score questionnaires.

\section{PSG Exclusion Criteria}

- $\quad$ Age $<18$ yrs.

- Very sick patients.

- Patients who are not willing for the study.

- Patients who did not complete their questionnaires and those who did not undergo PSG or did not complete their PSG study were excluded from the present study.

Sampling Methodology/ Population of the Study

The study was conducted over 62 patients in the Sleep Disorders Laboratory of Pulmonary Medicine, Sri Ramachandra University Hospital, Porur, Chennai.

\section{Period of Sampling}

The sample was taken into study for a period of 2 years from July 2015 - July 2017.

\section{Statistical Analysis}

Statistical analysis was performed utilising statistical package for social science software with IBM SPSS 23.0 version. Frequency analysis and percentage analysis was used for describing the categorical data. The mean and standard deviation was used to describe continuous data. To assess the efficacy of the tools (BERLIN, STOP-BANG, EPWORTH with AHI), the sensitivity, specificity, NPV (Negative Predicted Value) and PPV (Positive Predicted Value) ROC Curve, Chisquare were used.

Among the 62 patients studied, the percentage of gender distribution is $59.7 \%$ of males and $40.3 \%$ of females.

The study population ranged from 20 years to 80 years. Maximum number of patients was in the age group of $40-60$ years (36 patients). The mean age of the study population was 53 years.

Out of a total of 62 patients there were 3, 11 and 48 patients in the normal, overweight and obese classes respectively.

A neck circumference greater than 17 inches for men and 16 inches for women is associated with an increased risk of obstructive sleep apnoea.

Among 62 patients, the neck circumference was positive in $30(48.3 \%)$ patients and negative in $32(51.6 \%)$ patients. 
In the AHI severity, 8 patients fell in the range of $1-5$ (normal), 15 patients fell in the range of $6-15$ (diagnosed with mild OSA), 13 patients fell in the range of $16-30$ (diagnosed with moderate) and 26 patients had a score higher than 30 (diagnosed with severe).

In Berlin questionnaire, out of 62 patients 49 had a high risk of OSA answering, while 13 had a low risk of OSA answering.

In the STOP-BANG questionnaire there were 62 patients who were positive for snoring, 50 patients who were positive for tiredness, 44 patients who were positive for observed apnoea, 16 patients who had positive blood pressure, 36 patients who had positive BMI, 42 patients who had positive age, 30 patients who had positive neck circumference and 37 patients were positive for gender.

All the patients had a high risk of OSA answering yes in STOP-BANG questionnaire.

Out of 62 patients 30 had a high risk of excessive daytime sleepiness ESS $>11$, while 32 had a low risk of excessive daytime sleepiness ESS $<11$.

Berlin Score* AHI Cross-Tabulation

\begin{tabular}{|c|c|c|c|}
\hline \multirow{2}{*}{ COUNT } & \multicolumn{2}{|c|}{ AHI } & \multirow{2}{*}{ TOTAL } \\
\cline { 2 - 3 } & + ve & - ve & \\
\hline BERLIN SCORE +ve & 45 & 4 & 49 \\
\hline BERLIN SCORE -ve & 9 & 4 & 13 \\
\hline TOTAL & $\mathbf{5 4}$ & $\mathbf{8}$ & $\mathbf{6 2}$ \\
\hline
\end{tabular}

\begin{tabular}{|c|c|}
\hline Sensitivity & 83.33333 \\
\hline Specificity & 50 \\
\hline PPV & 91.83673 \\
\hline NPV & 30.76923 \\
\hline
\end{tabular}

The sensitivity was found to be 83.33 , specificity was found to be 50, PPV was found to be 91.84 and NPV was found to be 30.77 .

STOP-BANG Score* AHI Cross-Tabulation

\begin{tabular}{|c|c|c|c|}
\hline \multirow{2}{*}{ Count } & \multicolumn{2}{|c|}{ AHI } & \multirow{2}{*}{ Total } \\
\cline { 2 - 3 } & $+\mathbf{v e}$ & - ve & \\
\hline STOP BANG SCORE +ve & 54 & 8 & 62 \\
\hline STOP BANG SCORE -ve & 0 & 0 & 0 \\
\hline Total & $\mathbf{5 4}$ & $\mathbf{8}$ & $\mathbf{6 2}$ \\
\hline
\end{tabular}

\begin{tabular}{|c|c|}
\hline Sensitivity & 100 \\
\hline Specificity & 0 \\
\hline PPV & 87.09677 \\
\hline NPV & $\# \mathrm{DIV} / 0 !$ \\
\hline
\end{tabular}

The sensitivity was found to be 100 , specificity was found to be 0 , PPV was found to be 87.09 and NPV was found to be 0 .

Epworth Score* AHI Cross-Tabulation

\begin{tabular}{|c|c|c|c|}
\hline \multirow{2}{*}{ COUNT } & \multicolumn{2}{|c|}{ AHI } & \multirow{2}{*}{ TOTAL } \\
\cline { 2 - 3 } & + ve & - ve & \\
\hline EPWORTH SCORE +ve & 27 & 3 & 30 \\
\hline EPWORTH SCORE -ve & 27 & 5 & 32 \\
\hline Total & $\mathbf{5 4}$ & $\mathbf{8}$ & $\mathbf{6 2}$ \\
\hline
\end{tabular}

\begin{tabular}{|c|c|}
\hline Sensitivity & 50 \\
\hline Specificity & 62.5 \\
\hline PPV & 90 \\
\hline NPV & 15.625 \\
\hline
\end{tabular}

The sensitivity was found to be 50 , specificity was found to be 62.5 , PPV was found to be 90 and NPV was found to be 15.63.

\section{RESULTS}

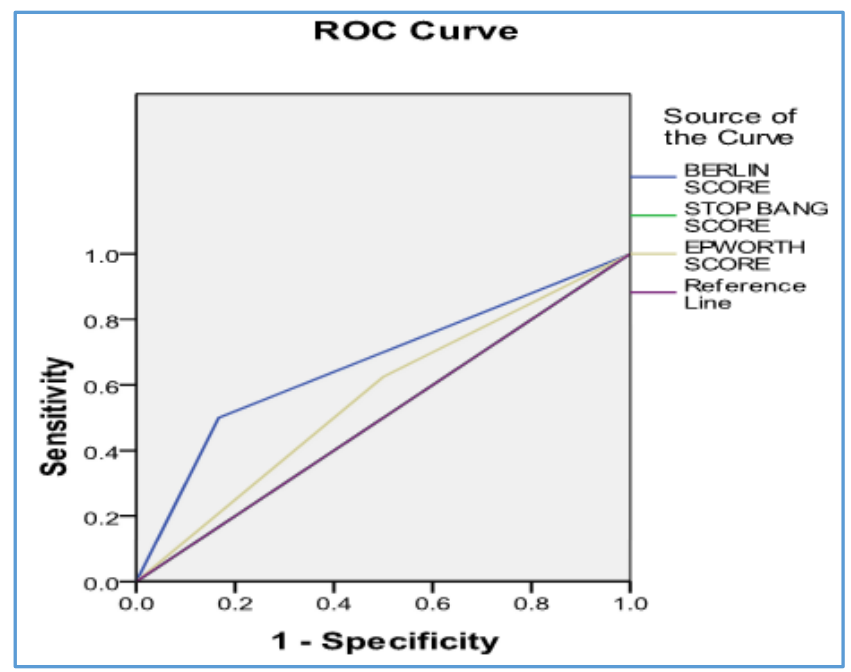

In the Berlin questionnaire, the average for sensitivity and specificity was found to be 66.65. In the STOP-BANG questionnaire, the average for sensitivity and specificity was found to be 50. In the Epworth questionnaire, the average for sensitivity and specificity was found to be 56.25 .

The above results show that the Berlin questionnaire score was better tool than the STOP-BANG and Epworth score, since the average accuracy of Berlin questionnaire was 66.65 .

\section{DISCUSSION}

This study aimed at comparing three established sleep questionnaires regarding their predictive probabilities for OSA. The questionnaires tested in this study were the STOPBANG and Berlin questionnaires as well as the ESS. All the questionnaires STOP-BANG, Berlin and Epworth were tested among the same population and the scores were evaluated against the PSG-based AHI serving as the "gold standard" diagnosis for OSA.

The cut-offs of the questionnaires used in this study were those previously published.

An ideal diagnostic test in a general population should have a relatively high specificity to minimise false positives, nevertheless, it should have sufficient sensitivity.

The STOP-BANG questionnaires were previously evaluated in some studies as preoperative screening instruments, mostly among the surgical population in an attempt to stratify patients for unrecognised OSA to prevent any possible OSA related intra- or post-operative complications.[12,13]

In an earlier study, it was found that the STOP-BANG questionnaire has high sensitivity for detecting OSA for moderate and severe OSA ( $93 \%$ and $100 \%$, respectively), yet the specificity at the same cut-off of the STOP-BANG questionnaire (score of P3) was still low: 47\% and 37\% for moderate and severe OSA, respectively, resulting in fairly high false-positive rates.

Another study reported that the STOP-BANG questionnaire had the highest sensitivity for moderate-to- 
severe $(87.0 \%)$ and severe SDB $(70.4 \%)$ in comparison to the ESS. STOP-BANG questionnaire had the advantage of being easily scored. Moreover, it was considered the most accurate questionnaires for OSA screening in surgical patients.[12]

The validity of our study was unclear, because patients were "pre-screened" for presence and frequency of snoring, wake-time sleepiness or fatigue and history of obesity or hypertension, which may have introduced selection bias.[7]

Based upon our study population results, the ESS had the lowest sensitivity to predict OSA in comparison to the other questionnaires. This is not surprising, because the ESS is a standard questionnaire to measure subjective excessive daytime sleepiness.[12]

Another study findings showed that there was an increase in the predictive parameters (namely sensitivity and NPV) of the ESS, Berlin, STOP-BANG questionnaires with the increase in severity of OSA, while the PPV decreased with the increase in severity for the three questionnaires.

In this study, the target population were patients presenting to the sleep clinic with sleep disorders. This might represent a potential for bias in the evaluation of the strength of different questionnaires to identify patients at risk for OSA owing to the fact that OSA is highly prevalent in "patients with sleep disorders." This can ultimately result in marked increase in the apparent sensitivity of the questionnaire and also reduces its specificity. In a recent systematic review Abrishami et al[7] reported that among "patients without history of sleep disorders" the Berlin questionnaire had the highest specificity, but the STOP-BANG questionnaires showed a lower specificity. Moreover, the Berlin questionnaire carried higher sensitivity in comparison to the STOP-BANG questionnaire carried the least sensitivity.

The comparison between the results of different studies concerned with the evaluation of sleep questionnaires is rather difficult based upon the following aspects; first, the PSG-based AHI cut-offs for OSA are not standardised in all studies, besides some studies use the RDI rather the AHI. Second, lack of a standard cut-off numbers are used for BMI in the questionnaires. Last but not the least, the studies are extremely diverse in their quality, design and the patient population.[7]

A key strength of our study is that all patients underwent a full-night attended diagnostic PSG, providing the "gold standard" against which the results of the questionnaires were compared. The questionnaires were answered prior to the PSG, which was in turn scored by the sleep specialist who was blinded to the results of the questionnaires and other clinical information concerning the patients to rule out any influence for the PSG over the results of the questionnaires. All questionnaires were tested among the same non-surgical population, yet the target population was patients presenting with sleep disorders.

In our study population of Berlin questionnaire, the average for sensitivity and specificity was found to be 66.65 ; in STOP-BANG questionnaire the average for sensitivity and specificity was found to be 50; and in Epworth questionnaire the average for sensitivity and specificity was found to be 56.25 .

\section{CONCLUSION}

- Aim of the study was to access the usefulness of Berlin, STOP-BANG and Epworth sleep questionnaires in evaluation of patient with OSA.

- The above results show that the Berlin questionnaires score is a better tool than the STOP-BANG and Epworth score, since the average accuracy of Berlin questionnaires was 66.65 .

- Large prospective studies will be required to help in establishing the exact role of subjective Berlin, STOPBANG and Epworth sleep screening questionnaires in evaluating patient with OSA.

\section{REFERENCES}

[1] Young T, Shahar E, Nieto FJ, et al. Predictors of sleepdisordered breathing in community-dwelling adults: the Sleep Heart Health Study. Arch Intern Med 2002;162(8):893-900.

[2] Young T, Palta M, Dempsey J, et al. The occurrence of sleep disordered breathing among middle-aged adults. N Engl J Med 1993;328(17):1230-5.

[3] Lettieri CJ, Eliasson AH, Andrada T, et al. Obstructive sleep apnea syndrome: are we missing an at-risk population? J Clin Sleep Med 2005;1(4):381-5.

[4] Young T, Evans L, Finn L, et al. Estimation of the clinically diagnosed proportion of sleep apnea syndrome in middle-aged men and women. Sleep 1997;20(9):705-6.

[5] Litvin AY, Sukmarova ZN, Elfimova EM, et al. Effects of CPAP on "vascular" risk factors in patients with obstructive sleep apnea and arterial hypertension. Vasc Health Risk Manag 2013;9:229-35.

[6] Noda A, Miyata S, Yasuda Y. Therapeutic strategies for sleep apnea in hypertension and heart failure. Article ID 814169, Pulm Med 2013;2013: p. 5.

[7] Abrishami A, Khajehdehi A, Chung F. A systematic review of screening questionnaires for obstructive sleep apnea. Can J Anaesth 2010;57(5):423-38.

[8] Deegan PC, McNicholas WT. Predictive value of clinical features for the obstructive sleep apnoea syndrome. Eur Respir J 1996;9(1):117-24.

[9] Ali NJ, Davies RJ, Fleetham JA, et al. Periodic movements of the legs during sleep associated with rises in systemic blood pressure. Sleep 1991;14(2):163-5.

[10] Crocker BD, Olson LG, Saunders NA, et al. Estimation of the probability of disturbed breathing during sleep before a sleep study. Am Rev Respir Dis 1990;142(1):14-8.

[11] Viner S, Szalai JP, Hoffstein V. Are history and physical examination a good screening test for sleep apnea? Ann Intern Med 1991;115(5):356-9.

[12] Chung F, Yegneswaran B, Liao P, et al. STOP questionnaire: a tool to screen patients for obstructive sleep apnea. Anesthesiology 2008;108(5):812-21.

[13] Bliwise DL, Nekich JC, Dement WC. Relative validity of self-reported snoring as a symptom of sleep apnea in a sleep clinic population. Chest 1991;99(3):600-8. 